TRANSACTIONS OF THE

AMERICAN MATHEMATICAL SOCIETY

Volume 363, Number 4, April 2011, Pages 1721-1741

S 0002-9947(2010)05094-9

Article electronically published on November 15, 2010

\title{
SUBORDINATED DISCRETE SEMIGROUPS OF OPERATORS
}

\author{
NICK DUNGEY
}

\begin{abstract}
Given a power-bounded linear operator $T$ in a Banach space and a probability $F$ on the non-negative integers, one can form a 'subordinated' operator $S=\sum_{k>0} F(k) T^{k}$. We obtain asymptotic properties of the subordinated discrete semigroup $\left(S^{n}: n=1,2, \ldots\right)$ under certain conditions on $F$. In particular, we study probabilities $F$ with the property that $S$ satisfies the Ritt resolvent condition whenever $T$ is power-bounded. Examples and counterexamples of this property are discussed. The hypothesis of power-boundedness of $T$ can sometimes be replaced by the weaker Kreiss resolvent condition.
\end{abstract}

\section{INTRODUCTION}

Let $\left(S_{t}\right)_{t \geq 0}$ be a uniformly bounded $C_{0}$ semigroup of operators on a complex Banach space $X$. Given a family $\left(\mu_{t}\right)_{t \geq 0}$ of probability measures on $\mathbb{R}^{+}:=[0, \infty)$ such that $\mu_{0}=\delta_{0}$ (the Dirac measure) and $\mu_{t_{1}} * \mu_{t_{2}}=\mu_{t_{1}+t_{2}}$, one can define a new semigroup $\left(\widehat{S}_{t}\right)_{t \geq 0}$ by

$$
\widehat{S}_{t}:=\int_{0}^{\infty} d \mu_{t}(s) S_{s}
$$

for $t \geq 0$. One says that the semigroup $\left(\widehat{S}_{t}\right)$ is subordinated to $\left(S_{t}\right)$ via the convolution semigroup $\left(\mu_{t}\right)_{t \geq 0}$. For more on this idea, which goes back to Bochner, see, for example, 4], [5, Section 2.4] and the references therein.

If $\left(\mu_{t}^{[\alpha]}\right)_{t \geq 0}$ is the convolution semigroup of so-called Lévy stable measures of order $\alpha \in(0,1)$, it is well known that the generator of $\left(\widehat{S}_{t}\right)$ is the fractional power $-A^{\alpha}$, where $-A$ is the generator of the semigroup $\left(S_{t}=e^{-t A}\right)_{t \geq 0}$. In this case a classic result of Yosida states that the semigroup $\left(\widehat{S}_{t}\right)_{t \geq 0}$ is always analytic; see, for example, 28, Section IX.11].

Subsequently Carasso and Kato 4 asked which convolution semigroups $\left(\mu_{t}\right)_{t \geq 0}$ on $\mathbb{R}^{+}$have the property that the subordinated semigroup is always analytic, and found various examples and counterexamples of this phenomenon.

The idea of the present paper is to develop an analogous theory for discrete time semigroups. Let $X$ be a complex Banach space and $T \in \mathcal{L}(X)$ be a bounded linear operator, which we usually assume to be power-bounded in the sense that $\sup _{n \in \mathbb{N}}\left\|T^{n}\right\|<\infty$, where $\mathbb{N}:=\{1,2,3, \ldots\}$. We consider $\left(T^{n}\right)_{n \in \mathbb{N}}$ as a discrete

Received by the editors January 27, 2008.

2000 Mathematics Subject Classification. Primary 47A30; Secondary 60G50, 47A60, 47D06.

Key words and phrases. Power-bounded operator, Ritt operator, discrete semigroup, analytic semigroup, subordinated semigroup.

(C)2010 American Mathematical Society Reverts to public domain 28 years from publication 
operator semigroup. Let $F$ be a probability on $\mathbb{Z}^{+}:=\{0,1,2, \ldots\}$; that is, $F: \mathbb{Z}^{+} \rightarrow$ $[0,1]$ satisfies $\sum_{k \geq 0} F(k)=1$. Setting $T^{0}:=I$, we define

$$
\Psi(F ; T):=\sum_{k \geq 0} F(k) T^{k} \in \mathcal{L}(X) .
$$

The powers $\Psi(F ; T)^{n}, n \in \mathbb{N}$, are then given by

$$
\Psi(F ; T)^{n}=\sum_{k \geq 0} F^{(n)}(k) T^{k}=\Psi\left(F^{(n)} ; T\right),
$$

where the probability $F^{(n)}$ is the $n$-th convolution power of $F$ (see Section 2 below for details). Since equation (3) is a discrete analogue of (11), we think of the discrete semigroup $\left(\Psi(F ; T)^{n}\right)_{n \in \mathbb{N}}$ as subordinate to $\left(T^{n}\right)$ via the probability $F$.

We are interested in regularity properties of the subordinated discrete semigroup $\left(\Psi(F ; T)^{n}\right)_{n \in \mathbb{N}}$. For example, in Section 3 we establish the time regularity property

$$
\sup _{n \in \mathbb{N}} n^{1 / 2}\left\|\Psi(F ; T)^{n}-\Psi(F ; T)^{n+1}\right\|<\infty
$$

whenever $T$ is power-bounded and $F$ satisfies a simple aperiodicity condition.

We will concentrate, however, on a stronger regularity property. Let us say that $S \in \mathcal{L}(X)$ is a Ritt operator if $S$ is power-bounded and

$$
\sup _{n \in \mathbb{N}} n\left\|S^{n}-S^{n+1}\right\|<\infty .
$$

This concept is a discrete time version of the notion of a bounded analytic $C_{0}$ semigroup, for it is well known (for example, [5, Section 2.5]) that a $C_{0}$ semigroup $\left(S_{t}=e^{-t A}\right)_{t \geq 0}$ is bounded analytic if and only if

$$
\sup _{t>0}\left(\left\|e^{-t A}\right\|+t\left\|A e^{-t A}\right\|\right)<\infty .
$$

A basic aim of the current paper is to study probabilities on $\mathbb{Z}^{+}$with the property that $\Psi(F ; T)$ is a Ritt operator for any power-bounded operator $T$. Fundamental examples are the probabilities $A_{\alpha}$ in the following theorem, which are connected with fractional powers of $I-T$ (see Section 4 for details).

Theorem 1.1. Let $\alpha \in(0,1)$. There exists a probability $A_{\alpha}$ on $\mathbb{Z}^{+}$such that for any complex Banach space $X$ and any power-bounded operator $T \in \mathcal{L}(X)$, the operator $\Psi\left(A_{\alpha} ; T\right)=I-(I-T)^{\alpha}$ is a Ritt operator.

Further interesting examples are the 'zeta' probabilities $Z_{\alpha}, \alpha \in(0,1)$. These are defined by $Z_{\alpha}(0)=0$ and

$$
Z_{\alpha}(k):=(\zeta(1+\alpha))^{-1} k^{-1-\alpha}
$$

for $k \in \mathbb{N}$, where $\zeta(s):=\sum_{n \geq 1} n^{-s}, s>1$, is the zeta function. We will establish the following result.

Theorem 1.2. For $\alpha \in(0,1)$ and any power-bounded operator $T \in \mathcal{L}(X)$, the operator $\Psi\left(Z_{\alpha} ; T\right)$ is a Ritt operator.

Theorems 1.1 and 1.2 are special cases in Section 4 where we give useful sufficient (though not necessary) conditions on a probability $F$ so that $\Psi(F ; T)$ is a Ritt operator whenever $T$ is power-bounded. Section 3 discusses some general properties of probabilities with the latter property. For example, such probabilities must have an infinite first moment, and therefore have rather slowly decaying tails. 
One can view the theory of large-time behaviour of random walks on $\mathbb{Z}^{+}$as behind many of our results. On the other hand, underlying the results of Carasso and Kato [4] is the rather different theory for small-time analysis of infinitely divisible convolution semigroups on $\mathbb{R}^{+}$. Thus some of our results on discrete subordinated semigroups have a quite different character from the continuous case studied in 4 .

Theorem 1.1 can be extended, by quite different methods, to operators $T$ satisfying a weaker condition than power-boundedness. Namely, we say that $T \in \mathcal{L}(X)$ is a Kreiss operator if it satisfies the Kreiss resolvent condition

$$
\left\|(\lambda I-T)^{-1}\right\| \leq c(|\lambda|-1)^{-1}
$$

for all $\lambda \in \mathbb{C}$ with $|\lambda|>1$. The expansion $(\lambda I-T)^{-1}=\sum_{n \geq 0} \lambda^{-1-n} T^{n}(|\lambda|>1)$ shows that every power-bounded operator is a Kreiss operator, but the converse is not true in infinite-dimensional Banach spaces (see, for example, 24, 21] and the references therein). In general, the convergence of the series (2) may be problematic when $T$ is a Kreiss operator. Nevertheless, in Section 6 we obtain the following connections between Kreiss operators, power-bounded operators and Ritt operators, by a different approach through the theory of fractional powers of operators.

Theorem 1.3. For $T \in \mathcal{L}(X)$, the following conditions are equivalent.

(I) $T$ is a Ritt operator.

(II) There exists a Kreiss operator $S$ and an $\alpha \in(0,1)$ such that $T=I-(I-S)^{\alpha}$. (III) There exists a power-bounded operator $S$ and an $\alpha \in(0,1)$ such that $T=$ $I-(I-S)^{\alpha}$.

(IV) $T$ is power-bounded, and there exists a $\gamma_{0}>1$ such that $I-(I-T)^{\gamma}$ is a Ritt operator for each $\gamma \in\left(1, \gamma_{0}\right)$.

The implication $(\mathrm{II}) \Rightarrow(\mathrm{I})$ in Theorem 1.3 partially generalizes Theorem 1.1 .

Note that condition (IV) in Theorem 1.3 gives a perturbation result for any Ritt operator $T$ under a type of 'fractional power' perturbation.

J. Zemánek asked whether there exist Ritt operators $T$ with $T \neq I$ and singlepoint spectrum $\sigma(T)=\{1\}$, a question answered affirmatively by Lyubich [19]. Our results actually reduce Zemánek's question to an easier question about Kreiss operators, as follows.

Corollary 1.4. Suppose that $T \in \mathcal{L}(X)$ is a Kreiss operator with $T \neq I$ and spectrum $\{1\}$. Then for each $\alpha \in(0,1)$, the operator $S:=I-(I-T)^{\alpha}$ is a Ritt operator with $S \neq I$ and spectrum $\{1\}$.

For example, consider the Volterra integral operator $V$ acting in $X=L^{2}(0,1)$, so $(V f)(x)=\int_{0}^{x} d t f(t)$ for $f \in L^{2}(0,1)$. It is well known that the operator $T:=$ $(I+V)^{-1}$ has spectrum $\{1\}$ and that $\|T\|=1$ (see [15, Problem 150], [21]). In particular, $T$ is power-bounded, so Corollary 1.4 applies and yields Ritt operators $I-(I-T)^{\alpha}, \alpha \in(0,1)$, each with spectrum $\{1\}$.

Actually, Lyubich's solution in [19] to Zemánek's question, which involves certain fractional Volterra-type operators, also seems to be a special case of Corollary 1.4 in view of the fact (see 21]) that the operator $T_{1}:=I-V$ is a Kreiss operator in the spaces $L^{p}(0,1), 1 \leq p \leq \infty$.

The following fundamental theorem on Ritt operators will be an important tool throughout the paper (for details of the theorem and further developments on Ritt operators, see [2, 3, 23, 24, 16, 27, 19] and their references). For $\theta \in(0, \pi)$ define the sectors $\Lambda_{\theta}:=\{z \in \mathbb{C}: z \neq 0,|\operatorname{Arg} z|<\theta\}$ and $\bar{\Lambda}_{\theta}:=\{0\} \cup\{z \in \mathbb{C}:|\operatorname{Arg} z| \leq \theta\}$. For 
$\theta=0$, we define $\bar{\Lambda}_{0}=[0, \infty)$. Put $\mathbb{D}:=\{z \in \mathbb{C}:|z|<1\}$ and $\overline{\mathbb{D}}:=\{z \in \mathbb{C}:|z| \leq 1\}$. In general $\sigma(T)$ denotes the spectrum of $T$.

Theorem 1.5. For $T \in \mathcal{L}(X)$, the following three conditions are equivalent.

(I) $T$ is a Ritt operator.

(II) One has $\sigma(T) \subseteq \mathbb{D} \cup\{1\}$, and the semigroup $\left(e^{-t(I-T)}\right)_{t \geq 0}$ is bounded analytic; that is,

$$
\sup _{t>0}\left(\left\|e^{-t(I-T)}\right\|+t\left\|(I-T) e^{-t(I-T)}\right\|\right)<\infty .
$$

(III) $T$ satisfies the Ritt resolvent condition; that is,

$$
\left\|(\lambda I-T)^{-1}\right\| \leq c|\lambda-1|^{-1}
$$

for all $\lambda \in \mathbb{C}$ with $|\lambda|>1$.

Moreover, if these conditions are satisfied, then there is a $\theta \in(0, \pi / 2)$ such that

$$
\sigma(T) \subseteq(\mathbb{D} \cup\{1\}) \cap\left\{z \in \mathbb{C}: 1-z \in \bar{\Lambda}_{\theta}\right\} .
$$

The final statement of Theorem 1.5 follows from condition (II), since for a bounded analytic semigroup $\left(e^{-t A}\right)_{t \geq 0}$ it is standard ([5, Section 2.5]) that $\sigma(A) \subseteq$ $\bar{\Lambda}_{\theta}$ for some $\theta \in(0, \pi / 2)$.

\section{Preliminaries}

This section establishes definitions and preliminary results for subordinated discrete semigroups. We refer to 6, 12 for standard material on Banach algebras and harmonic analysis, and to [10, 13] for Fourier (and other) transforms of probability measures.

Suppose that $\mathcal{B}$ is a complex Banach algebra with unit element $1_{\mathcal{B}}$. The spectrum of an element $A \in \mathcal{B}$ is written $\sigma(A ; \mathcal{B})$; in case $\mathcal{B}=\mathcal{L}(X)$, the algebra of bounded operators in a complex Banach space $X$, this is abbreviated to $\sigma_{X}(A)$ or $\sigma(A)$. For $A \in \mathcal{B}$ the exponential is $e^{A}:=\sum_{k \geq 0}(k !)^{-1} A^{k} \in \mathcal{B}$, where $A^{0}:=1_{\mathcal{B}}$. One says that $A$ is power-bounded if $\sup _{n \in \mathbb{N}}\left\|A^{n}\right\|<\infty$. If $A$ is power-bounded, then $\sigma(A ; \mathcal{B}) \subseteq \overline{\mathbb{D}}$.

The Banach algebra $L^{1}(\mathbb{Z})$ consists of all functions $F: \mathbb{Z} \rightarrow \mathbb{C}$ on the integers $\mathbb{Z}$ such that $\|F\|_{L^{1}(\mathbb{Z})}:=\sum_{k \in \mathbb{Z}}|F(k)|<\infty$; the (commutative) convolution product $F_{1} * F_{2}$ of $F_{1}, F_{2} \in L^{1}(\mathbb{Z})$ is given by

$$
\left(F_{1} * F_{2}\right)(m)=\sum_{k \in \mathbb{Z}} F_{1}(k) F_{2}(m-k), \quad m \in \mathbb{Z} .
$$

Write $F^{(n)}:=F * F * \cdots * F$ for the $n$-th convolution power of $F \in L^{1}(\mathbb{Z})$. The unit element of $L^{1}(\mathbb{Z})$ is $\delta_{0}$, where in general $\delta_{m} \in L^{1}(\mathbb{Z})$ is defined so that $\delta_{m}(k)$ is 1 or 0 according to whether $k=m$ or $k \neq m, k, m \in \mathbb{Z}$.

For $F \in L^{1}(\mathbb{Z})$ define the $\mathbb{Z}$-Fourier transform $\widehat{F} \in C([-\pi, \pi])$ by $\widehat{F}(\xi)=$ $\sum_{k \in \mathbb{Z}} F(k) e^{-i k \xi}, \xi \in[-\pi, \pi]$. Consider the convolution operator $L(F) \in \mathcal{L}\left(L^{1}(\mathbb{Z})\right)$ given by $L(F) F_{1}:=F * F_{1}, F_{1} \in L^{1}(\mathbb{Z})$. Then $L: L^{1}(\mathbb{Z}) \rightarrow \mathcal{L}\left(L^{1}(\mathbb{Z})\right)$ is an algebra homomorphism, with

and

$$
\|L(F)\|_{\mathcal{L}\left(L^{1}(\mathbb{Z})\right)}=\|F\|_{L^{1}(\mathbb{Z})}
$$

$$
\sigma_{L^{1}(\mathbb{Z})}(L(F))=\sigma\left(F ; L^{1}(\mathbb{Z})\right)=\{\widehat{F}(\xi): \xi \in[-\pi, \pi]\}
$$

for all $F \in L^{1}(\mathbb{Z})$. For $F \in L^{1}(\mathbb{Z})$ define the $\operatorname{support} \operatorname{supp}(F):=\{k \in \mathbb{Z}: F(k) \neq$ $0\}$. 
Put

$$
L^{1}\left(\mathbb{Z}^{+}\right):=\left\{F \in L^{1}(\mathbb{Z}): \operatorname{supp}(F) \subseteq \mathbb{Z}^{+}=\{0,1,2, \ldots\}\right\} ;
$$

then $L^{1}\left(\mathbb{Z}^{+}\right)$is a closed subalgebra of $L^{1}(\mathbb{Z})$. For $F \in L^{1}\left(\mathbb{Z}^{+}\right)$, define $\phi_{F}: \overline{\mathbb{D}} \rightarrow \mathbb{C}$ by

$$
\phi_{F}(w):=\sum_{k \geq 0} F(k) w^{k}, \quad w \in \overline{\mathbb{D}} .
$$

Clearly $\phi_{F}$ is continuous on $\overline{\mathbb{D}}$ and analytic in $\mathbb{D},\left\|\phi_{F}\right\|_{L^{\infty}(\overline{\mathbb{D}})} \leq\|F\|_{L^{1}(\mathbb{Z})}$, and $\widehat{F}$ gives the boundary values of $\phi_{F}$ in the sense that $\phi_{F}\left(e^{-i \xi}\right)=\widehat{F}(\xi)$ for $\xi \in[-\pi, \pi]$. Moreover $\phi_{F_{1} * F_{2}}=\phi_{F_{1}} \phi_{F_{2}}$ for all $F_{1}, F_{2} \in L^{1}\left(\mathbb{Z}^{+}\right)$.

Let $\mathbb{P}(\mathbb{Z}):=\left\{F \in L^{1}(\mathbb{Z}): F \geq 0, \sum_{k \in \mathbb{Z}} F(k)=1\right\}$ be the set of probabilities on $\mathbb{Z}$. A probability $F \in \mathbb{P}(\mathbb{Z})$ is said to be adapted if $\operatorname{supp}(F)$ generates the additive group $\mathbb{Z}$. As a stronger condition, $F \in \mathbb{P}(\mathbb{Z})$ is said to be aperiodic if all of the translated probabilities $\delta_{m} * F, m \in \mathbb{Z}$, are adapted. It is a useful remark that $F \in \mathbb{P}(\mathbb{Z})$ is aperiodic whenever $\operatorname{supp}(F)$ contains two consecutive integers $k, k+1$.

To each $F \in \mathbb{P}(\mathbb{Z})$ we associate a continuous convolution semigroup $\left(e^{-t\left(\delta_{0}-F\right)}\right)_{t>0}$ $\subseteq \mathbb{P}(\mathbb{Z})$ of probabilities. Note that the formula $e^{-t\left(\delta_{0}-F\right)}=e^{-t} \sum_{n \geq 0}(n !)^{-1} t^{n} F^{(n)}$ shows that these are indeed probabilities.

Set $\mathbb{P}\left(\mathbb{Z}^{+}\right):=\mathbb{P}(\mathbb{Z}) \cap L^{1}\left(\mathbb{Z}^{+}\right)$. For $F \in \mathbb{P}\left(\mathbb{Z}^{+}\right)$, the function $\phi_{F}: \overline{\mathbb{D}} \rightarrow \overline{\mathbb{D}}$ is called the 'generating function' of $F$ in classical probability theory.

Given a power-bounded operator $T \in \mathcal{L}(X)$ and any $F \in L^{1}\left(\mathbb{Z}^{+}\right)$, define $\Psi(F ; T)$ $\in \mathcal{L}(X)$ by equation (2). Clearly $\Psi(F ; T)$ depends linearly on $F$, and

$$
\|\Psi(F ; T)\| \leq c(T)\|F\|_{L^{1}(\mathbb{Z})},
$$

where $c(T):=\sup _{k \geq 0}\left\|T^{k}\right\|$. One has $\Psi\left(F_{1} * F_{2} ; T\right)=\Psi\left(F_{1} ; T\right) \Psi\left(F_{2} ; T\right)$ for all $F_{1}, F_{2} \in L^{1}\left(\mathbb{Z}^{+}\right)$. This yields equation (3) for any $F \in L^{1}\left(\mathbb{Z}^{+}\right)$.

For $F \in L^{1}\left(\mathbb{Z}^{+}\right)$it is natural to write ' $\Psi(F ; T)=\phi_{F}(T)$ ', but we will not use the functional calculus notation $\phi_{F}(T)$ systematically.

For any $T \in \mathcal{L}(X)$ (not necessarily power-bounded) and any function $\varphi$ analytic on a neighborhood of $\sigma(T)$, the operator $\varphi(T) \in \mathcal{L}(X)$ is defined by the Dunford functional calculus, and the spectral mapping theorem (see, for example, [28, Section VIII.7]) states that $\sigma(\varphi(T))=\varphi(\sigma(T))$. For power-bounded $T$ we have $\sigma(T) \subseteq \overline{\mathbb{D}}$; the next result extends the spectral mapping theorem to $\Psi(F ; T)=\phi_{F}(T)$ for any $F \in L^{1}\left(\mathbb{Z}^{+}\right)$(observe that $\phi_{F}$ need not be analytic on a neighborhood of $\overline{\mathbb{D}})$.

Theorem 2.1. Let $T \in \mathcal{L}(X)$ be power-bounded. Then one has $\sigma(\Psi(F ; T))=$ $\phi_{F}(\sigma(T)) \subseteq \phi_{F}(\overline{\mathbb{D}})$ for all $F \in L^{1}\left(\mathbb{Z}^{+}\right)$.

Proof. Given $F \in L^{1}\left(\mathbb{Z}^{+}\right)$, choose a sequence $\left(F_{n}\right)_{n \in \mathbb{N}} \subseteq L^{1}\left(\mathbb{Z}^{+}\right)$such that $\lim _{n \rightarrow \infty}\left\|F_{n}-F\right\|_{L^{1}(\mathbb{Z})}=0$ and each $F_{n}$ has finite support. Since $\Psi\left(F_{n} ; T\right)$ is a polynomial in $T$, the above-cited spectral mapping theorem yields $\sigma\left(\Psi\left(F_{n} ; T\right)\right)=$ $\phi_{F_{n}}(\sigma(T))$ for all $n$. A standard spectral perturbation result ([18, Theorem IV.3.6]) states that for any commuting operators $S_{1}, S_{2} \in \mathcal{L}(X)$ one has

$$
\rho\left(\sigma\left(S_{1}\right), \sigma\left(S_{2}\right)\right) \leq\left\|S_{1}-S_{2}\right\|,
$$

where

$$
\rho\left(\Gamma_{1}, \Gamma_{2}\right):=\max \left(\sup _{x \in \Gamma_{1}} \inf _{y \in \Gamma_{2}}|x-y|, \sup _{y \in \Gamma_{2}} \inf _{x \in \Gamma_{1}}|x-y|\right)
$$


is the Hausdorff distance between two compact sets $\Gamma_{1}, \Gamma_{2} \subseteq \mathbb{C}$. Then since

$$
\lim _{n \rightarrow \infty}\left\|\Psi\left(F_{n} ; T\right)-\Psi(F ; T)\right\|=0
$$

by (6), we deduce that

$$
\lim _{n \rightarrow \infty} \rho\left(\phi_{F_{n}}(\sigma(T)), \sigma(\Psi(F ; T))\right)=0 .
$$

But also $\lim _{n \rightarrow \infty} \rho\left(\phi_{F_{n}}(\sigma(T)), \phi_{F}(\sigma(T))\right)=0$, as a consequence of the fact that $\lim _{n \rightarrow \infty}\left\|\phi_{F_{n}}-\phi_{F}\right\|_{L^{\infty}(\overline{\mathbb{D}})}=0$. By uniqueness of limits for the Hausdorff metric we obtain $\sigma(\Psi(F ; T))=\phi_{F}(\sigma(T))$.

The following basic observation is a consequence of (3) and (6) and will sometimes be used without comment.

Lemma 2.2. Let $T \in \mathcal{L}(X)$ be power-bounded. If $F \in L^{1}\left(\mathbb{Z}^{+}\right)$is power-bounded (that is, $\left.\sup _{n \in \mathbb{N}}\left\|F^{(n)}\right\|_{L^{1}(\mathbb{Z})}<\infty\right)$, then $\Psi(F ; T) \in \mathcal{L}(X)$ is power-bounded. In particular, $\Psi(F ; T)$ is power-bounded for each $F \in \mathbb{P}\left(\mathbb{Z}^{+}\right)$.

In this section we establish a basic regularity estimate for the differences $\Psi(F ; T)^{n}-\Psi(F ; T)^{n+1}$.

We first quote a result which characterizes a decay of order $O\left(n^{-1 / 2}\right)$ of the differences $\left\|S^{n}-S^{n+1}\right\|$, for an $S \in \mathcal{L}(X)$; note that this decay is slower than for Ritt operators.

Theorem 2.3. Given $S \in \mathcal{L}(X)$, the following two conditions are equivalent.

(I) The operator $S$ is power-bounded, and $\sup _{n \in \mathbb{N}} n^{1 / 2}\left\|S^{n}-S^{n+1}\right\|<\infty$.

(II) There exist $\beta \in(0,1)$ and a power-bounded operator $T \in \mathcal{L}(X)$ such that $S=\beta T+(1-\beta) I$.

If these conditions hold, then there is a $\beta \in(0,1)$ such that

$$
\sigma(S) \subseteq\{z \in \mathbb{C}:|z-(1-\beta)| \leq \beta\} \subseteq \mathbb{D} \cup\{1\} .
$$

The implication $(\mathrm{II}) \Rightarrow(\mathrm{I})$ in Theorem 2.3 is contained in [11, Lemma 2.1], 222, Theorem 4.5.3] or [24, Theorem 8]. The implication (I) $\Rightarrow(\mathrm{II})$ was recently proved by the author (see [8, where a number of other equivalent conditions on $S$ are studied). However, strictly speaking we do not need the latter implication in the present paper.

The statement about $\sigma(S)$ in Theorem 2.3 is a consequence of condition (II) and the fact that $\sigma(T) \subseteq \overline{\mathbb{D}}$ for power-bounded $T$.

In Theorem 2.3(II) we have $S=\Psi(F ; T)$, where $F \in \mathbb{P}\left(\mathbb{Z}^{+}\right)$is the 'Bernoulli' probability with $\operatorname{supp}(F)=\{0,1\}, F(0)=1-\beta, F(1)=\beta$. The main result of this section, stated next, generalizes the implication $(\mathrm{II}) \Rightarrow(\mathrm{I})$ in Theorem 2.3 to arbitrary aperiodic probabilities.

Theorem 2.4. Let $T \in \mathcal{L}(X)$ be power-bounded, and let $F \in \mathbb{P}\left(\mathbb{Z}^{+}\right)$be aperiodic. Then

$$
\sup _{n \in \mathbb{N}} n^{1 / 2}\left\|\Psi(F ; T)^{n}-\Psi(F ; T)^{n+1}\right\|<\infty,
$$

and there exists a $\beta \in(0,1)$ such that

$$
\sigma(\Psi(F ; T)) \subseteq\{z \in \mathbb{C}:|z-(1-\beta)| \leq \beta\} \subseteq \mathbb{D} \cup\{1\} .
$$


To prove Theorem 2.4 we require the following result proved in the Appendix (Section 7 below). The equivalence of conditions (I) and (II) below is known (see [26. Chapter II], where the term 'strongly aperiodic' is used to mean aperiodic). However, to our knowledge, condition (IV) seems to be new and interesting.

Proposition 2.5. Let $F \in \mathbb{P}(\mathbb{Z})$ be adapted. Then the following conditions are equivalent.

(I) $F$ is aperiodic.

(II) $|\widehat{F}(\xi)|<1$ for all $\xi \in[-\pi, \pi] \backslash\{0\}$.

(III) $\widehat{F}(\xi) \in \mathbb{D} \cup\{1\}$ for all $\xi \in[-\pi, \pi]$.

(IV) There exist $\beta \in(0,1)$ and a power-bounded element $G \in L^{1}(\mathbb{Z})$ (that is, $\left.\sup _{n \in \mathbb{N}}\left\|G^{(n)}\right\|_{L^{1}(\mathbb{Z})}<\infty\right)$ such that $F=\beta G+(1-\beta) \delta_{0}$.

If these conditions hold, then

$$
\sup _{n \in \mathbb{N}} n^{1 / 2}\left\|F^{(n)}-F^{(n+1)}\right\|_{L^{1}(\mathbb{Z})}<\infty .
$$

Proof of Theorem 2.4. Choose $\beta$ and $G$ as in part (IV) of Proposition 2.5. Then $G \in L^{1}\left(\mathbb{Z}^{+}\right)$, the operator $\Psi(G ; T)$ is power-bounded, and $\Psi(F ; T)=\beta \Psi(G ; T)+$ $(1-\beta) I$. Theorem 2.4 therefore follows from Theorem 2.3 .

An alternative way to obtain (7) is to write

$$
\Psi(F ; T)^{n}-\Psi(F ; T)^{n+1}=\Psi\left(F^{(n)}-F^{(n+1)} ; T\right)
$$

and apply (8) together with (6).

Remark. If $F \in \mathbb{P}(\mathbb{Z})$ satisfies $F(0)>0$, then condition (IV) of Proposition 2.5 is easy to obtain (and aperiodicity is not needed). In fact, it suffices to choose $\beta \in(1-F(0), 1)$ and observe that

$$
G:=\beta^{-1}\left(F-(1-\beta) \delta_{0}\right)
$$

is an element of $\mathbb{P}(\mathbb{Z})$. However, if $F(0)=0$ (a case we shall often encounter), then $G$ in condition (IV) cannot be a probability.

\section{The Class $\mathcal{A}$}

In this section we begin our study of the class $\mathcal{A}=\mathcal{A}\left(\mathbb{Z}^{+}\right) \subseteq \mathbb{P}\left(\mathbb{Z}^{+}\right)$of 'Ritt' probabilities, which we define by

$$
\mathcal{A}:=\left\{F \in \mathbb{P}\left(\mathbb{Z}^{+}\right): \sup _{n \in \mathbb{N}} n\left\|F^{(n)}-F^{(n+1)}\right\|_{L^{1}(\mathbb{Z})}<\infty\right\} .
$$

Clearly $\delta_{0} \in \mathcal{A}$; non-trivial examples of probabilities in $\mathcal{A}$ will be constructed in Sections 4 and [5. In this section we develop general properties of the class $\mathcal{A}$, including its connection with subordinated discrete semigroups.

Here is our basic characterization of the class $\mathcal{A}$. It was partly inspired by the results on continuous convolution semigroups in [4, Theorem 4].

Theorem 3.1. Given $F \in \mathbb{P}\left(\mathbb{Z}^{+}\right)$, the following four conditions are equivalent.

(I) $F \in \mathcal{A}$.

(II) The operator $L(F)$ is a Ritt operator in $L^{1}(\mathbb{Z})$.

(III) For any complex Banach space $X$ and any power-bounded operator $T \in \mathcal{L}(X)$, the operator $\Psi(F ; T)$ is a Ritt operator.

(IV) One has $\widehat{F}(\xi) \in \mathbb{D} \cup\{1\}$ for all $\xi \in[-\pi, \pi]$, and

$$
\sup _{t \geq 1} t\left\|\left(\delta_{0}-F\right) * e^{-t\left(\delta_{0}-F\right)}\right\|_{L^{1}(\mathbb{Z})}<\infty .
$$


Moreover, if $F \in \mathcal{A}$, then there is a $\theta \in(0, \pi / 2)$ such that

$$
\phi_{F}(w) \in \mathbb{D} \cup\{1\}, \quad 1-\phi_{F}(w) \in \bar{\Lambda}_{\theta}
$$

for all $w \in \overline{\mathbb{D}}$. Finally, if $F \in \mathcal{A} \backslash\left\{\delta_{0}\right\}$, then

$$
\sum_{k \geq 0} k F(k)=+\infty
$$

and hence $\operatorname{supp}(F)$ is infinite.

Proof. (I) $\Leftrightarrow(\mathrm{II})$ : observe that

$$
\begin{gathered}
\left\|L(F)^{n}-L(F)^{n+1}\right\|_{\mathcal{L}\left(L^{1}(\mathbb{Z})\right)}=\left\|F^{(n)}-F^{(n+1)}\right\|_{L^{1}(\mathbb{Z})} . \\
(\mathrm{I}) \Rightarrow(\mathrm{III}) \text { : setting } c(T):=\sup _{k \geq 0}\left\|T^{k}\right\|, \text { one has } \\
\left\|\Psi(F ; T)^{n}-\Psi(F ; T)^{n+1}\right\|=\left\|\Psi\left(F^{(n)}-F^{(n+1)} ; T\right)\right\| \leq c(T)\left\|F^{(n)}-F^{(n+1)}\right\|_{L^{1}(\mathbb{Z})}
\end{gathered}
$$

for all $n \in \mathbb{N}$, where the last step used (6). that

$(\mathrm{III}) \Rightarrow(\mathrm{II})$ : consider the operator $T:=L\left(\delta_{1}\right)$ acting in $X=L^{1}(\mathbb{Z})$ and observe

$$
L(F)=\sum_{k \geq 0} F(k) T^{k}=\Psi(F ; T) .
$$

$(\mathrm{IV}) \Leftrightarrow(\mathrm{II})$ : recalling (5) , one sees that condition (IV) holds if and only if $L(F) \in$ $\mathcal{L}\left(L^{1}(\mathbb{Z})\right)$ satisfies the conditions $\sigma(L(F)) \subseteq \mathbb{D} \cup\{1\}$ and

$$
\sup _{t \geq 1} t\left\|(I-L(F)) e^{-t(I-L(F))}\right\|<\infty .
$$

By Theorem 1.5 those conditions hold if and only if $L(F)$ is a Ritt operator in $L^{1}(\mathbb{Z})$.

Thus we have shown the equivalence of all the conditions (I)-(IV).

We next assume that $F \in \mathcal{A}$ and establish (9). Consider the Banach space $X=C(\overline{\mathbb{D}})$ of continuous functions on $\overline{\mathbb{D}}$ under the usual supremum norm, and let $T \in \mathcal{L}(X)$ be the multiplication operator $(T f)(w):=w f(w)$ for $f \in C(\overline{\mathbb{D}}), w \in \overline{\mathbb{D}}$. Then $\|T\|=1$, and $\Psi(F ; T)$ is the multiplication operator

$$
(\Psi(F ; T) f)(w)=\phi_{F}(w) f(w), \quad w \in \overline{\mathbb{D}} .
$$

By applying the final statement of Theorem 1.5 to the Ritt operator $\Psi(F ; T)$, since $\sigma(\Psi(F ; T))=\phi_{F}(\overline{\mathbb{D}})$ we obtain (9).

To prove the last assertion (10), suppose that $F \in \mathbb{P}\left(\mathbb{Z}^{+}\right), F \neq \delta_{0}$, and $a:=$ $\sum_{k>0} k F(k)<\infty$; we will show that $F \notin \mathcal{A}$. Note first that $a>0$. Differentiating $\widehat{F}(\xi)=\sum_{k>0} F(k) e^{-i k \xi}$ with respect to $\xi$ shows that $\widehat{F} \in C^{1}([-\pi, \pi])$ and $\widehat{F}^{\prime}(0)=$ $-i a$. Since $\widehat{F}(0)=1$ we have

$$
\widehat{F}(\xi)=1-i a \xi+o(|\xi|),
$$

where $o(|\xi|)$ denotes a function of $\xi \in[-\pi, \pi]$ such that $\lim _{\xi \rightarrow 0} o(|\xi|) /|\xi|=0$. Since $a>0$, for some small $\varepsilon \in(0,1)$ we have $|1-\widehat{F}(\xi)| \geq \varepsilon|\xi|$ for all $\xi \in[-\varepsilon, \varepsilon]$, while $1-\operatorname{Re} \widehat{F}(\xi)=o(|\xi|)$. Because $\widehat{F}(\xi)=\phi_{F}\left(e^{-i \xi}\right)$, it follows that the second statement of (9) cannot hold. Therefore $F \notin \mathcal{A}$. 
Remarks. For the Laplace transforms of certain continuous convolution semigroups on $\mathbb{R}^{+}$, 4, Theorem 4] gives a sectorial condition somewhat analogous to the second part of (9).

Although we believe that the conditions (9) are not sufficient for a probability $F$ to be in $\mathcal{A}$, it does not seem easy to find counterexamples.

Regarding (10), for $F \in \mathbb{P}(\mathbb{Z})$ the quantity $\sum_{k \in \mathbb{Z}} k F(k)$ (when it exists) is the first moment or 'center of mass' of $F$. On any compactly generated locally compact group (in particular on $\mathbb{Z}$ ) there is a large class of 'Ritt' probability measures which have finite, in fact vanishing, first moments; see [7] for this theory. On $\mathbb{Z}^{+}$, however, the only probability with a vanishing first moment is $\delta_{0}$.

Condition (10) shows that many commonly studied probabilities on $\mathbb{Z}^{+}$ (for example, probabilities of finite support, or the Poisson probability $P_{s}:=e^{-s} \sum_{k>0}(k !)^{-1} s^{k} \delta_{k}$ for any $\left.s>0\right)$ are not elements of $\mathcal{A}$.

In Section 5 we shall describe an example satisfying (10) but not (9).

Here are some further interesting properties of the class $\mathcal{A}$.

Proposition 3.2. The set $\mathcal{A}$ is a convex subset of $\mathbb{P}\left(\mathbb{Z}^{+}\right)$. For any $F_{1}, F_{2} \in \mathcal{A}$ and $G \in \mathbb{P}\left(\mathbb{Z}^{+}\right)$, one has $F_{1} * F_{2} \in \mathcal{A}$ and

$$
\sum_{k \geq 0} F_{1}(k) G^{(k)} \in \mathcal{A}
$$

Proof. Let $F_{1}, F_{2} \in \mathcal{A}$. From

$$
\left(F_{1} * F_{2}\right)^{(n)}-\left(F_{1} * F_{2}\right)^{(n+1)}=\left(F_{1}^{(n)}-F_{1}^{(n+1)}\right) * F_{2}^{(n)}+F_{1}^{(n+1)} *\left(F_{2}^{(n)}-F_{2}^{(n+1)}\right)
$$

it easily follows that $F_{1} * F_{2} \in \mathcal{A}$. For a convex combination $F=\lambda F_{1}+(1-\lambda) F_{2} \in$ $\mathbb{P}\left(\mathbb{Z}^{+}\right)$, where $\lambda \in(0,1)$, one has

$$
e^{-t\left(\delta_{0}-F\right)}=e^{-t \lambda\left(\delta_{0}-F_{1}\right)} * e^{-t(1-\lambda)\left(\delta_{0}-F_{2}\right)} .
$$

By differentiating this formula with respect to $t$ and applying Condition (IV) of Theorem 3.1 one sees that $F \in \mathcal{A}$. Thus $\mathcal{A}$ is convex. Finally, consider the probability $H:=\sum_{k>0} F_{1}(k) G^{(k)} \in \mathbb{P}\left(\mathbb{Z}^{+}\right)$. Since $L(H)=\Psi\left(F_{1} ; L(G)\right)$ and $F_{1} \in$ $\mathcal{A}$, we deduce from Theorem 3.1(III) that $L(H)$ is a Ritt operator in $L^{1}(\mathbb{Z})$; hence $H \in \mathcal{A}$.

It follows from Theorem 3.1(IV) and Proposition 2.5 that an element of $\mathcal{A}$ is aperiodic if and only if it is adapted. The next result shows that non-aperiodic elements of $\mathcal{A}$ can be obtained by scaling of aperiodic elements. Thus in practice it suffices to study the aperiodic elements of $\mathcal{A}$.

Lemma 3.3. Suppose that $F \in \mathcal{A} \backslash\left\{\delta_{0}\right\}$ is not aperiodic. Then there exist $m \in$ $\{2,3,4, \ldots\}$ and an aperiodic probability $\widetilde{F} \in \mathcal{A}$ such that $\operatorname{supp}(F) \subseteq\{0, m, 2 m, \ldots\}$ and $F(k m)=\widetilde{F}(k)$ for all $k \in \mathbb{Z}^{+}$.

Proof. By the remark preceding the lemma, $F$ is not adapted. Since $F \neq \delta_{0}$, the subgroup of $\mathbb{Z}$ generated by $\operatorname{supp}(F)$ must equal $m \mathbb{Z}$ for some $m \in\{2,3, \ldots\}$. Set $\widetilde{F}(k):=F(k m)$ for $k \in \mathbb{Z}^{+}$. Then $\widetilde{F} \in \mathbb{P}\left(\mathbb{Z}^{+}\right)$is adapted, and $\widetilde{F} \in \mathcal{A}$ because $\left\|\widetilde{F}^{(n)}-\widetilde{F}^{(n+1)}\right\|_{L^{1}(\mathbb{Z})}=\left\|F^{(n)}-F^{(n+1)}\right\|_{L^{1}(\mathbb{Z})}$ for all $n \in \mathbb{N}$. By the remark preceding the lemma, $\widetilde{F}$ is aperiodic. 


\section{SUfFicient CONDITIONS AND EXAMPLES}

In this section we first give a sufficient criterion for a probability to belong to $\mathcal{A}$, in terms of the $\mathbb{Z}$-Fourier transform. This result leads to fundamental examples of elements of $\mathcal{A}$, including the probabilities $A_{\alpha}, Z_{\alpha}$ in Theorems 1.1 and 1.2 .

Write $\partial_{\xi}$ for differentiation with respect to the real variable $\xi$.

Theorem 4.1. Let $\alpha \in(0,1)$.

(I) Suppose that $F \in \mathbb{P}\left(\mathbb{Z}^{+}\right)$is aperiodic and that there exist $\varepsilon \in(0,1), c>0$ such that

$$
1-\operatorname{Re} \widehat{F}(\xi) \geq \varepsilon|\xi|^{\alpha}
$$

for all $\xi \in[-\varepsilon, \varepsilon]$ and

$$
\left|\partial_{\xi} \widehat{F}(\xi)\right| \leq c|\xi|^{\alpha-1}
$$

for all $\xi \in[-\pi, \pi] \backslash\{0\}$. Then $F \in \mathcal{A}$.

(II) Let $F, G \in \mathbb{P}\left(\mathbb{Z}^{+}\right)$be aperiodic with $F$ satisfying the conditions of part (I) above. If there exists an $a>0$ such that

$$
\sum_{k \geq 1} k|G(k)-a F(k)|<\infty,
$$

then $G$ satisfies the conditions of part (I) above, and hence $G \in \mathcal{A}$.

Part (I) above is partly inspired by an argument of Carasso and Kato dealing with Fourier transforms of Lévy stable measures (see 4, Example 3]).

Proof of Theorem 4.1. To prove part (I), we will verify condition (IV) of Theorem [3.1. By Proposition 2.5 one has $\widehat{F}(\xi) \subseteq \mathbb{D} \cup\{1\}$ for all $\xi \in[-\pi, \pi]$. In fact if $\delta=\sup \{\operatorname{Re} \widehat{F}(\xi):|\xi| \in\langle\varepsilon, \pi]\}$, then $\delta<1$ (see Section 7). Hence $1-\operatorname{Re} \widehat{F}(\xi) \geq \pi^{-\alpha}(1-\delta)|\xi|^{\alpha}$ for $|\xi| \in\langle\varepsilon, \pi]$. So by modifying $\varepsilon$ we may assume that (12) holds for all $\xi \in[-\pi, \pi]$.

Set $G_{t}:=\left(\delta_{0}-F\right) * e^{-t\left(\delta_{0}-F\right)} \in L^{1}\left(\mathbb{Z}^{+}\right)$for each $t>0$. Put $R_{t}:=\widehat{G_{t}}$, so that

$$
R_{t}(\xi)=(1-\widehat{F}(\xi)) e^{-t(1-\widehat{F}(\xi))} .
$$

Integrating (13) yields a bound $|1-\widehat{F}(\xi)|=|\widehat{F}(0)-\widehat{F}(\xi)| \leq c^{\prime}|\xi|^{\alpha}$ for $\xi \in[-\pi, \pi]$. Using this together with (12), one finds that there are $c, b>0$ such that

$$
\begin{aligned}
\left|R_{t}(\xi)\right| & =|1-\widehat{F}(\xi)| e^{-t(1-\operatorname{Re} \widehat{F}(\xi))} \leq c|\xi|^{\alpha} e^{-b t|\xi|^{\alpha}}, \\
\left|\partial_{\xi} R_{t}(\xi)\right| & \leq c|\xi|^{\alpha-1} e^{-b t|\xi|^{\alpha}}
\end{aligned}
$$

for all $t \geq 1$ and $\xi \in[-\pi, \pi]$. Note that $\partial_{\xi} R_{t}$ is the $\mathbb{Z}$-Fourier transform of the function $H_{t}(k):=-i k G_{t}(k), k \in \mathbb{Z}$.

The next part of the argument is similar to [4, pp. 875-876]. Fix $r>1$ with $r<\min \left\{2,(1-\alpha)^{-1}\right\}$, and set $s:=r /(r-1) \in(2, \infty)$. Let $\|\cdot\|_{r}$ denote the norm in the space $L^{r}([-\pi, \pi] ; d \xi)$. Integrating (14) gives bounds of the type

$$
\left\|R_{t}\right\|_{r} \leq c t^{-1} t^{-1 /(\alpha r)}, \quad\left\|\partial_{\xi} R_{t}\right\|_{r} \leq c t^{-1} t^{1 /(\alpha s)}
$$

for all $t \geq 1$. The inverse $\mathbb{Z}$-Fourier transform is a bounded operator from $L^{r}([-\pi, \pi])$ into $L^{s}(\mathbb{Z})$, by the Hausdorff-Young inequality ([25, Theorem 12.12]); hence

$$
\left\|G_{t}\right\|_{L^{s}(\mathbb{Z})} \leq c^{\prime} t^{-1} t^{-1 /(\alpha r)}, \quad\left\|H_{t}\right\|_{L^{s}(\mathbb{Z})} \leq c^{\prime} t^{-1} t^{1 /(\alpha s)}
$$


for $t \geq 1$. Using Hölder's inequality we obtain

$$
\begin{aligned}
\left\|G_{t}\right\|_{L^{1}(\mathbb{Z})} \leq & \left(\sum_{k \geq 0}\left(1+k t^{-1 / \alpha}\right)^{-r}\right)^{1 / r} \\
& \times\left(\sum_{k \geq 0}\left|G_{t}(k)\right|^{s}\left(1+k t^{-1 / \alpha}\right)^{s}\right)^{1 / s} \\
\leq & c t^{1 /(\alpha r)}\left[\left\|G_{t}\right\|_{L^{s}(\mathbb{Z})}+t^{-1 / \alpha}\left\|H_{t}\right\|_{L^{s}(\mathbb{Z})}\right] \leq c^{\prime} t^{-1}
\end{aligned}
$$

for all $t \geq 1$. Thus $F$ satisfies Condition (IV) of Theorem 3.1 and $F \in \mathcal{A}$.

To prove part (II), put $P:=G-a F \in L^{1}\left(\mathbb{Z}^{+}\right)$. The hypothesis $\sum_{k>1} k|P(k)|<$ $\infty$ implies that $\widehat{P} \in C^{1}([-\pi, \pi])$. Since $\widehat{G}(0)=\widehat{F}(0)=1$ one has $\widehat{P}(0)=1-a$, and hence $|\widehat{P}(\xi)-(1-a)| \leq c^{\prime}|\xi|$ for all $\xi \in[-\pi, \pi]$. The equations

$$
1-\widehat{G}=a(1-\widehat{F})-(\widehat{P}-(1-a)), \quad \partial_{\xi} \widehat{G}(\xi)=a \partial_{\xi} \widehat{F}(\xi)+\partial_{\xi} \widehat{P}(\xi)
$$

then show that $G$ satisfies estimates of types (12) and (13).

Our first application of Theorem 4.1, to probabilities $A_{\alpha}, \alpha \in(0,1)$, is based on the following lemma. Write

$$
\left(\begin{array}{c}
\beta \\
k
\end{array}\right):=(k !)^{-1} \beta(\beta-1) \cdots(\beta-(k-1))
$$

for $\beta \in \mathbb{R}$ and $k \in \mathbb{N}$. For $\beta>0$ we always consider the principal branch of the power function $z \mapsto z^{\beta}$ defined for $z \in \mathbb{C} \backslash(-\infty, 0)$.

Lemma 4.2. Given $\alpha \in(0,1)$, set

$$
a_{k}=a_{k}^{(\alpha)}:=(-1)^{k-1}\left(\begin{array}{c}
\alpha \\
k
\end{array}\right)=\frac{\alpha(1-\alpha)(2-\alpha) \cdots((k-1)-\alpha)}{k !}
$$

for $k \in \mathbb{N}$. Then $a_{k}>0$ for all $k \in \mathbb{N}, \sum_{k \geq 1} a_{k}=1$, and

$$
1-(1-w)^{\alpha}=\sum_{k \geq 1} a_{k} w^{k}
$$

for all $w \in \overline{\mathbb{D}}$. In addition,

$$
a_{k}=\alpha \Gamma(1-\alpha)^{-1} k^{-\alpha-1}+O\left(k^{-\alpha-2}\right)
$$

for $k \in \mathbb{N}$.

Proof. The binomial series expansion

$$
(1-w)^{\alpha}=1+\sum_{k \geq 1}\left(\begin{array}{c}
\alpha \\
k
\end{array}\right)(-1)^{k} w^{k}
$$

is valid for all $w \in \mathbb{D}$, giving (16) for $w \in \mathbb{D}$. The last expression in (15) shows that $a_{k}>0$. For any $r \in(0,1)$ and $N \in \mathbb{N}$ we have

$$
\sum_{k=1}^{N} a_{k} r^{k} \leq \sum_{k \geq 1} a_{k} r^{k}=1-(1-r)^{\alpha}<1 .
$$


Taking limits as $r \rightarrow 1$ and then as $N \rightarrow \infty$ yields $\sum_{k \geq 1} a_{k} \leq 1$. Thus each side of (16) defines a continuous function of $w \in \overline{\mathbb{D}}$, so by continuity (16) holds for all $w \in \overline{\mathbb{D}}$. Taking $w=1$ in (16) yields $\sum_{k \geq 1} a_{k}=1$.

As a preliminary step to (17), we claim that

$$
a_{k} \sim \alpha \Gamma(1-\alpha)^{-1} k^{-\alpha-1}, \quad k \rightarrow \infty,
$$

where the notation $\sim$ means that the ratio of the two sides converges to 1 as $k \rightarrow \infty$. Now

$$
a_{k}=\frac{\alpha((k-1)-\alpha)((k-2)-\alpha) \cdots(1-\alpha) \Gamma(1-\alpha)}{k ! \Gamma(1-\alpha)}=\frac{\alpha \Gamma(k-\alpha)}{\Gamma(1-\alpha) \Gamma(k+1)}
$$

and from Stirling's formula $\Gamma(t+1) \sim(2 \pi t)^{1 / 2} t^{t} e^{-t}, t \rightarrow \infty$, one has

$$
\begin{aligned}
\frac{\Gamma(k-\alpha)}{\Gamma(k+1)} & \sim \frac{(k-\alpha-1)^{k-\alpha-1+(1 / 2)} e^{-(k-\alpha-1)}}{k^{k+(1 / 2)} e^{-k}} \\
& =e^{\alpha+1}\left(1-(\alpha+1) k^{-1}\right)^{k+(1 / 2)}(k-\alpha-1)^{-\alpha-1} \\
& \sim(k-\alpha-1)^{-\alpha-1} \sim k^{-\alpha-1}
\end{aligned}
$$

from which (18) follows. Using the precise Stirling's estimate

$$
\left|\frac{\Gamma(t+1)}{(2 \pi t)^{1 / 2} t^{t} e^{-t}}-1\right| \leq c t^{-1}, \quad t \geq 1,
$$

it is straightforward to refine the proof of (18) to get (17); we leave this to the reader.

Applying Lemma 4.2, for each $\alpha \in(0,1)$ define $A_{\alpha} \in \mathbb{P}\left(\mathbb{Z}^{+}\right)$by $A_{\alpha}(0):=0$ and

$$
A_{\alpha}(k):=a_{k}^{(\alpha)}=\frac{\alpha(1-\alpha)(2-\alpha) \cdots((k-1)-\alpha)}{k !}
$$

for $k \in \mathbb{N}$. Lemma 4.2 implies that $A_{\alpha}$ has the generating function

$$
\phi_{A_{\alpha}}(w)=1-(1-w)^{\alpha} .
$$

Given a power-bounded operator $T$, it is therefore natural to write $\Psi\left(A_{\alpha} ; T\right)=$ $I-(I-T)^{\alpha}$. Since

$$
\widehat{A}_{\alpha}(\xi)=1-\left(1-e^{-i \xi}\right)^{\alpha}
$$

for $\xi \in[-\pi, \pi]$, one easily checks that $F=A_{\alpha}$ satisfies the conditions of Theorem 4.1(I), so that $A_{\alpha} \in \mathcal{A}$. These observations and Theorem 3.1yield Theorem 1.1, which we now restate more precisely.

Theorem 4.3. For each $\alpha \in(0,1)$, the probability $A_{\alpha} \in \mathbb{P}\left(\mathbb{Z}^{+}\right)$defined by (19) is an element of $\mathcal{A}$. For any power-bounded operator $T \in \mathcal{L}(X)$, the operator $\Psi\left(A_{\alpha} ; T\right)=I-(I-T)^{\alpha}$ is a Ritt operator.

We next consider the zeta probabilities $Z_{\alpha} \in \mathbb{P}\left(\mathbb{Z}^{+}\right)$defined by (4).

Theorem 4.4. For each $\alpha \in(0,1)$ one has $Z_{\alpha} \in \mathcal{A}$.

Observe that Theorem 4.4 yields Theorem 1.2 ,

Proof of Theorem 4.4. We mention two methods of proof. The first method is to observe from (17) that there is a constant $c_{\alpha}>0$ with

$$
Z_{\alpha}(k)=c_{\alpha} A_{\alpha}(k)+O\left(k^{-2-\alpha}\right), \quad k \in \mathbb{N} .
$$


Applying Theorem 4.1(II) with $F=A_{\alpha}, G=Z_{\alpha}$ shows that $Z_{\alpha} \in \mathcal{A}$. In fact $A_{\alpha}(k)=\alpha \Gamma(1-\alpha)^{-1} k^{-1-\alpha}+O\left(k^{-2-\alpha}\right)$ by (17) and, in addition, $Z_{\alpha}(k)=$ $(\zeta(1+\alpha))^{-1} k^{-1-\alpha}$ by (4), so $c_{\alpha}=\zeta(1+\alpha) \Gamma(1-\alpha)^{-1}$.

The second method involves the polylogarithm $\mathrm{Li}_{s}$, which is defined by

$$
\operatorname{Li}_{s}(w):=\sum_{k \geq 1} k^{-s} w^{k}
$$

for $s>1, w \in \overline{\mathbb{D}}$, and can be analytically continued in $w$ to a function analytic on $\mathbb{C} \backslash[1, \infty)$. For $s>1$ with $s \notin \mathbb{N}$, one has a series expansion

$$
\operatorname{Li}_{s}\left(e^{\mu}\right)=\zeta(s)+\Gamma(1-s)(-\mu)^{s-1}+\sum_{n \geq 1} \zeta(s-n)(n !)^{-1} \mu^{n}
$$

for all $\mu \in \mathbb{C}$ with $|\mu|<2 \pi, \mu \notin(0,2 \pi)$. This result is contained in the expansion of the special function $\Phi$ in 9 , p. 29, equation (8)], noting that $\operatorname{Li}_{\mathrm{s}}(w)=w \Phi(w, s, 1)$. Taking $s=1+\alpha$ and $\mu=-i \xi$ in (21) one obtains

$$
\begin{aligned}
\widehat{Z}_{\alpha}(\xi) & =\zeta(1+\alpha)^{-1} \operatorname{Li}_{1+\alpha}\left(e^{-i \xi}\right) \\
& =1-b_{\alpha}(i \xi)^{\alpha}+\xi \varphi_{\alpha}(\xi)
\end{aligned}
$$

for all $\xi \in[-\pi, \pi]$, where $b_{\alpha}:=-\Gamma(-\alpha) \zeta(1+\alpha)^{-1}>0$ and where $\varphi_{\alpha} \in C^{\infty}([-\pi, \pi])$ is a smooth function. It readily follows that $F=Z_{\alpha}$ satisfies the conditions of Theorem 4.1(I), so $Z_{\alpha} \in \mathcal{A}$.

The next result considerably generalizes the examples $Z_{\alpha}$ and $A_{\alpha}$.

Theorem 4.5. Suppose that $F \in \mathbb{P}\left(\mathbb{Z}^{+}\right)$is such that

$$
F(k)=\sum_{j=1}^{N} c_{j} k^{-1-\alpha_{j}}+P(k)
$$

for all $k \in \mathbb{N}$, where $N \in \mathbb{N}, 0<\alpha_{1}<\cdots<\alpha_{N}<1, c_{1}, \ldots, c_{N}>0$, and $P \in$ $L^{1}\left(\mathbb{Z}^{+}\right)$with $\sum_{k \geq 1} k|P(k)|<\infty$. Then $F$ satisfies the conditions of Theorem 4.1 (I) with $\alpha=\alpha_{1}$. Therefore $F \in \mathcal{A}$.

Proof. It is easy to see that $\operatorname{supp}(F)$ must contain two consecutive integers $k, k+1$, and therefore $F$ is aperiodic. Set $\widetilde{c}:=\sum_{j=1}^{N} c_{j} \zeta\left(1+\alpha_{j}\right)>0, \lambda_{j}:=\widetilde{c}^{-1} c_{j} \zeta\left(1+\alpha_{j}\right) \in$ $(0,1)$, and define

$$
G:=\sum_{j=1}^{N} \lambda_{j} Z_{\alpha_{j}} \in \mathbb{P}\left(\mathbb{Z}^{+}\right),
$$

a convex combination of the probabilities $Z_{\alpha_{j}}$. It is straightforward to see that $G$ satisfies the conditions of Theorem 4.1(I) with $\alpha=\alpha_{1}$. Because $F=\widetilde{c} G+P+$ $[F(0)-P(0)] \delta_{0}$, the theorem follows by applying Theorem 4.1(II).

We end this section with a formula connecting $A_{\alpha}$ with the convolution semigroup $\left(\mu_{t}^{[\alpha]}\right)_{t \geq 0}$ of Lévy stable probability measures on $\mathbb{R}^{+}$. For fixed $\alpha \in(0,1)$, the measures $\mu_{t}^{[\alpha]}, t \geq 0$, are implicitly defined by their Laplace transforms:

$$
\int_{0}^{\infty} d \mu_{t}^{[\alpha]}(s) e^{-s z}=e^{-t z^{\alpha}}
$$


for all $z \in \mathbb{C}$ with $\operatorname{Re} z \geq 0$ (for details, see [28, Section IX.11], [10, Chapter XIII] and [4). Let $\left(P_{s}\right)_{s \geq 0} \subseteq \mathbb{P}\left(\mathbb{Z}^{+}\right)$be the Poisson convolution semigroup, defined by

$$
P_{s}:=e^{-s\left(\delta_{0}-\delta_{1}\right)}=e^{-s} \sum_{k \geq 0}(k !)^{-1} s^{k} \delta_{k} .
$$

Proposition 4.6. Let $\alpha \in(0,1)$. One has the equality

$$
e^{-t\left(\delta_{0}-A_{\alpha}\right)}=\int_{0}^{\infty} d \mu_{t}^{[\alpha]}(s) P_{s}
$$

as elements of $\mathbb{P}\left(\mathbb{Z}^{+}\right)$, for all $t \geq 0$.

Roughly speaking, $\left(e^{-t\left(\delta_{0}-A_{\alpha}\right)}\right)_{t>0}$ is the convolution semigroup subordinate to the Poisson semigroup via $\left(\mu_{t}^{[\alpha]}\right)_{t \geq 0}$.

Proof of Proposition 4.6. Fix $t>0$. Each side of (22) defines an element of $\mathbb{P}\left(\mathbb{Z}^{+}\right)$, and it suffices to show that both sides have the same generating function. Because $P_{s}$ has generating function $w \mapsto e^{-s(1-w)}$, the right side of (22) has generating function

$$
w \mapsto \int_{0}^{\infty} d \mu_{t}^{[\alpha]}(s) e^{-s(1-w)}=e^{-t(1-w)^{\alpha}} .
$$

From (20) one sees that the left side of (22) also has generating function $e^{-t(1-w)^{\alpha}}$.

\section{Further EXAMPLES}

We first construct some examples of elements of $\mathcal{A}$ which do not fall under the hypotheses of Theorem 4.1. In these examples $1-\phi_{F}(w)$ has a type of logarithmic decay as $w \rightarrow 1$ within the disc $\overline{\mathbb{D}}$.

Theorem 5.1. Fix $\varepsilon \in(0,1]$ and set

$$
B:=\varepsilon^{-1} \int_{0}^{\varepsilon} d \alpha A_{\alpha} \in \mathbb{P}\left(\mathbb{Z}^{+}\right)
$$

(with $A_{\alpha}$ defined by (19)). For each $\beta \in(0,1)$ put $B_{\beta}:=\sum_{k \geq 0} A_{\beta}(k) B^{(k)}$. Then $B_{\beta} \in \mathcal{A}$, and

$$
\lim _{\xi \rightarrow 0} \frac{|\xi|^{\alpha}}{\left|1-\widehat{B_{\beta}}(\xi)\right|}=0
$$

for each $\alpha \in(0,1)$.

Integration of the estimate (13) yields a bound $|1-\widehat{F}(\xi)| \leq c^{\prime}|\xi|^{\alpha}$ for all $\xi \in$ $[-\pi, \pi]$; thus (24) shows that the probability $F=B_{\beta}$ cannot satisfy (13) for $\alpha \in$ $(0,1)$.

We point out that (23) has a formal similarity to a construction of 19] (see also [16, Section 2]), where a Ritt operator is produced by integrating certain fractional Volterra operators.

Proof of Theorem 5.1. Clearly (23) defines an element $B \in \mathbb{P}\left(\mathbb{Z}^{+}\right)$. Since $A_{\beta} \in \mathcal{A}$, Proposition 3.2 implies that $B_{\beta} \in \mathcal{A}$.

Using (20) one computes

$$
1-\phi_{B}(w)=\varepsilon^{-1} \int_{0}^{\varepsilon} d \alpha(1-w)^{\alpha}=\varepsilon^{-1}[\log (1-w)]^{-1}\left((1-w)^{\varepsilon}-1\right)
$$


for $w \in \overline{\mathbb{D}}$; here $\log : \mathbb{C} \backslash(-\infty, 0] \rightarrow \mathbb{C}$ is the principal branch of the logarithm, with the additional convention $(\log (0))^{-1}=0$. Replacing $w$ by $e^{-i \xi}$ in (25), because of the logarithmic factor one sees that

$$
\lim _{\xi \rightarrow 0} \frac{|\xi|^{\alpha}}{|1-\widehat{B}(\xi)|}=0
$$

for each $\alpha \in(0,1)$. But the definition of $B_{\beta}$ implies that

$$
\phi_{B_{\beta}}(w)=\sum_{k \geq 0} A_{\beta}(k)\left(\phi_{B}(w)\right)^{k}=\phi_{A_{\beta}}\left(\phi_{B}(w)\right)=1-\left(1-\phi_{B}(w)\right)^{\beta} ;
$$

hence $1-\widehat{B_{\beta}}(\xi)=(1-\widehat{B}(\xi))^{\beta}$, and (24) follows.

We conjecture that $B$ defined by (23) is an element of $\mathcal{A}$, for any $\varepsilon \in(0,1$. Evidence for this conjecture is that the generating function $\phi_{B}$ satisfies the sectorial condition in (9), as is easily verified from the first relation in (25). Actually, $\phi_{B}$ (and similarly $\phi_{B_{\beta}}$ ) satisfies not only (9) but also

$$
\lim _{w \in \mathbb{\mathbb { D }}, w \rightarrow 1} \operatorname{Arg}\left(1-\phi_{B}(w)\right)=0 .
$$

This contrasts with the probabilities $A_{\alpha}, \alpha \in(0,1)$, which satisfy (use (201)

$$
\limsup _{w \in \overline{\mathbb{D}}, w \rightarrow 1}\left|\operatorname{Arg}\left(1-\phi_{A_{\alpha}}(w)\right)\right|=\alpha \pi / 2 .
$$

The construction of Theorem 5.1 can be iterated to produce further examples of elements of $\mathcal{A}$. For example, if for fixed $\varepsilon \in(0,1], \beta \in(0,1)$ we set

$$
C:=\varepsilon^{-1} \int_{0}^{\varepsilon} d \alpha B_{\alpha}, \quad C_{\beta}:=\sum_{k \geq 0} A_{\beta}(k) C^{(k)},
$$

then $C_{\beta} \in \mathcal{A}$, and $1-\phi_{C_{\beta}}(w)=\left(1-\phi_{C}(w)\right)^{\beta}$ has a type of iterated logarithmic decay (slower than logarithmic decay) as $w \rightarrow 1$. We leave the details to the reader.

We next give an example in a quite different direction.

Theorem 5.2. There exists an aperiodic probability $F \in \mathbb{P}\left(\mathbb{Z}^{+}\right)$satisfying $\sum_{k \geq 0} k F(k)=+\infty$ (that is, (10) ) such that

$$
\lim _{\xi \rightarrow 0}|\operatorname{Arg}(1-\widehat{F}(\xi))|=\pi / 2 \text {. }
$$

Thus $F$ does not satisfy (91), so $F \notin \mathcal{A}$.

Proof. Define $F \in L^{1}\left(\mathbb{Z}^{+}\right)$by $F(0)=F(1)=0$ and

$$
F(k)=\frac{1}{k(k-1)}
$$

for $k \in\{2,3,4, \ldots\}$. Clearly $\sum_{k \geq 0} k F(k)=+\infty$. Using the expansion $\log (1-w)=$ $-\sum_{k \geq 1} k^{-1} w^{k}$ one checks that

$$
1-\phi_{F}(w)=1-\sum_{k \geq 2} \frac{w^{k}}{k(k-1)}=(1-w)[1-\log (1-w)]
$$

for all $w \in \overline{\mathbb{D}} \backslash\{1\}$. Taking the limit as $w \rightarrow 1$ yields $\phi_{F}(1)=1$, so $F \in \mathbb{P}\left(\mathbb{Z}^{+}\right)$. By setting $w=e^{-i \xi}$ in the second relation for $1-\phi_{F}(w)$ one easily verifies (26).

Similarly, it is possible to show that the probability $Z_{1}(k):=\zeta(2)^{-1} k^{-2}, k \in \mathbb{N}$ (which is the case $\alpha=1$ of (44)) satisfies the statements of Theorem 5.2. 


\section{KREISS OPERATORS AND FRACTIONAL POWERS}

The aim of this section is to establish a generalization of Theorem 1.1 for Kreiss operators, and then to prove Theorem 1.3. Our approach is based on the theory of fractional powers of linear operators (cf. 20]) and is essentially independent of the theory of previous sections. In particular, we do not use the idea of subordination via probabilities.

We need some preliminaries. A densely defined, closed linear operator $V$ in the complex Banach space $X$ is said to be of type $\omega$, where $\omega \in[0, \pi)$, if $\sigma(V) \subseteq \bar{\Lambda}_{\omega}$ and

$$
\sup _{\lambda \in \Lambda_{\pi-\omega-\varepsilon}}\left\|\lambda(\lambda I+V)^{-1}\right\|<\infty
$$

for every $\varepsilon \in(0, \pi-\omega)$. It is a standard fact ([5, Section 2.5]) that $V$ is of type $\omega$ for some $\omega \in[0, \pi / 2)$ if and only if $-V$ is the generator of a bounded analytic semigroup $\left(e^{-t V}\right)_{t \geq 0}$ whose angle is $\frac{\pi}{2}-\omega$.

For an operator $V$ of type $\omega$ the fractional powers $V^{\alpha}$ can be defined for all $\alpha>0$; see [14, 20] for recent expositions of this theory. One has the relations $V^{\alpha} V^{\beta}=$ $V^{\alpha+\beta}$ for $\alpha, \beta>0$, and $\left(V^{\alpha}\right)^{\beta}=V^{\alpha \beta}$ for $\alpha \in(0,1), \beta>0$. The spectral mapping theorem for fractional powers states that

$$
\sigma\left(V^{\alpha}\right)=\left\{z^{\alpha}: z \in \sigma(V)\right\}, \quad \alpha>0 .
$$

If, in addition, the operator $V$ is bounded, then $V^{\alpha}$ is bounded for $\alpha>0$.

The next result appears, for example, in [1, Corollary 3.10], [14, Proposition 3.1.2], and in case $\alpha \in(0,1)$ in [17. Since this result is important for our purposes, we sketch a proof.

Theorem 6.1. Let $V$ be of type $\omega \in(0, \pi)$. Then for each $\alpha \in(0, \pi / \omega)$ the operator $V^{\alpha}$ is of type $\omega \alpha$.

Proof. By the formula $V^{\beta}=\left(V^{\beta / 2^{n}}\right)^{2^{n}}$ for $\beta>0, n \in \mathbb{N}$ with $\beta<2^{n}$, the proof of the theorem easily reduces to the two cases $\alpha \in(0,1)$ and $\alpha=2$.

In case $\alpha \in(0,1)$ we use the resolvent formula of Kato [17],

$$
\left(\lambda I+V^{\alpha}\right)^{-1}=\frac{\sin \alpha \pi}{\pi} \int_{0}^{\infty} d t t^{\alpha}\left[\left(\lambda+e^{i \alpha \pi} t^{\alpha}\right)\left(\lambda+e^{-i \alpha \pi} t^{\alpha}\right)\right]^{-1}(t I+V)^{-1},
$$

where the integral converges for each $\lambda \in \Lambda_{(1-\alpha) \pi}$. For $V$ of type $\omega$ and $|\theta|<\pi-\omega$, replace $V$ by $e^{-i \theta} V$ in this formula; one can then estimate the norm of the operator $\left(\lambda I+e^{-i \alpha \theta} V^{\alpha}\right)^{-1}=e^{i \alpha \theta}\left(\lambda e^{i \alpha \theta} I+V^{\alpha}\right)^{-1}$ for $\lambda \in \Lambda_{(1-\alpha) \pi}$ to see that $V^{\alpha}$ is of type $\alpha \omega$.

For $V$ of type $\omega \in(0, \pi / 2)$, one can see that $V^{2}$ is of type $2 \omega$ via the identity

$$
\left(t e^{i \theta} I+V^{2}\right)^{-1}=\left(i t^{1 / 2} e^{i \theta / 2} I+V\right)^{-1}\left(-i t^{1 / 2} e^{i \theta / 2} I+V\right)^{-1}
$$

valid for $t>0$ and $|\theta|<\pi-2 \omega$.

Theorem 6.2. Suppose that $T \in \mathcal{L}(X)$ is a Kreiss operator. Then $(I-T)^{\alpha}$ is of type $\alpha \pi / 2$ for all $\alpha \in(0,1]$, and $I-(I-T)^{\alpha}$ is a Ritt operator for all $\alpha \in(0,1)$.

Proof. One has $|\mu+1|-1 \geq \operatorname{Re} \mu$ for all $\mu \in \mathbb{C}$. Therefore the Kreiss resolvent condition implies that

$$
\left\|(\mu I+(I-T))^{-1}\right\| \leq c(\operatorname{Re} \mu)^{-1}
$$


whenever $\operatorname{Re} \mu>0$. It follows that $I-T$ is of type $\pi / 2$, and hence $(I-T)^{\alpha}$ is of type $\alpha \pi / 2$ for $\alpha \in(0,1)$. Thus for $\alpha \in(0,1)$, the operator $S:=I-(I-T)^{\alpha} \in \mathcal{L}(X)$ is such that $\left(e^{-t(I-S)}\right)_{t \geq 0}$ is a bounded analytic semigroup. Also, from $\sigma(T) \subseteq \overline{\mathbb{D}}$ and the spectral mapping theorem (27) one obtains $\sigma(S) \subseteq\left\{1-(1-z)^{\alpha}: z \in \overline{\mathbb{D}}\right\} \subseteq$ $\mathbb{D} \cup\{1\}$. By Theorem 1.5, $S$ is a Ritt operator.

Remark. The hypothesis in Theorem 6.2 that $T$ be Kreiss can be considerably weakened: the conclusions of the theorem hold whenever $T \in \mathcal{L}(X)$ is such that $I-T$ is of type $\pi / 2$ and $\sigma(T) \subseteq \overline{\mathbb{D}}$.

The next result is a corollary of Theorem 6.1

Corollary 6.3. If $-V$ is the generator of a bounded analytic semigroup $\left(e^{-t V}\right)_{t \geq 0}$, then there exists $\gamma_{0}>1$ such that $-V^{\gamma}$ is the generator of a bounded analytic semigroup $\left(e^{-t V^{\gamma}}\right)_{t \geq 0}$ for each $\gamma \in\left(1, \gamma_{0}\right)$.

Proof. Because $V$ is of type $\omega$ for some $\omega \in(0, \pi / 2)$, then $V^{\gamma}$ is of type $\omega \gamma<\pi / 2$ whenever $\gamma \in(1, \pi /(2 \omega))$.

Proof of Theorem 1.3 . (IV) $\Rightarrow$ (III) is proved by setting $S:=I-(I-T)^{\gamma}$ for some $\gamma \in\left(1, \gamma_{0}\right)$, noting that $I-T=(I-S)^{1 / \gamma}$. (III) $\Rightarrow$ (II) is immediate since every power-bounded operator is a Kreiss operator. (II $\Rightarrow(\mathrm{I})$ is just Theorem 6.2

Let us prove $(\mathrm{I}) \Rightarrow(\mathrm{IV})$. Because $T$ is Ritt the semigroup $\left(e^{-t(I-T)}\right)_{t \geq 0}$ is bounded analytic as a consequence of Theorem 1.5, and Corollary 6.3 implies that the semigroup $\left(e^{-t(I-T)^{\gamma}}\right)_{t \geq 0}$ is bounded analytic for $\gamma>1$ sufficiently close to 1 . Also, by (27) and by the last statement of Theorem 1.5 applied to $\sigma(T)$, it is easy to see that

$$
\sigma\left(I-(I-T)^{\gamma}\right)=\left\{1-(1-z)^{\gamma}: z \in \sigma(T)\right\} \subseteq \mathbb{D} \cup\{1\}
$$

for $\gamma>1$ sufficiently close to 1 . Thus by Theorem 1.5 the operator $I-(I-T)^{\gamma}$ is a Ritt operator for $\gamma>1$ sufficiently close to 1 .

Finally, Corollary 1.4 is a consequence of Theorem 6.2 the spectral mapping (27), and the observation that $(I-T)^{\alpha} \neq 0$ whenever $\alpha \in(0,1)$ and $T \neq I$. (For, if $(I-T)^{\alpha}=0$, then $I-T=\left((I-T)^{\alpha}\right)^{1 / \alpha}=0$.)

\section{Appendix}

In this Appendix we give a proof of Proposition 2.5. Throughout we consider an adapted probability $F \in \mathbb{P}(\mathbb{Z})$. Observe the properties $\widehat{F}(0)=1$ and $|\widehat{F}(\xi)| \leq 1$ for all $\xi \in[-\pi, \pi]$.

$(\mathrm{I}) \Rightarrow(\mathrm{II})$ : suppose that $F$ is aperiodic and $\eta \in[-\pi, \pi]$ with $|\widehat{F}(\eta)|=1$; we must show that $\eta=0$. Setting $\tau:=\widehat{F}(\eta)=\sum_{k \in \mathbb{Z}} F(k) e^{-i k \eta}$, since $|\tau|=1$ it follows that

$$
e^{-i k \eta}=\tau
$$

for all $k \in \operatorname{supp}(F)$. After fixing a $k^{\prime} \in \operatorname{supp}(F)$, then $e^{-i\left(k-k^{\prime}\right) \eta}=1$ so that $\eta\left(k-k^{\prime}\right) \in 2 \pi \mathbb{Z}:=\{2 \pi m: m \in \mathbb{Z}\}$ for all $k \in \operatorname{supp}(F)$. Aperiodicity implies that the set $\left\{k-k^{\prime}: k \in \operatorname{supp}(F)\right\}$ generates the group $\mathbb{Z}$. Therefore $\eta \mathbb{Z} \subseteq 2 \pi \mathbb{Z}$, so that $\eta \in 2 \pi \mathbb{Z}$ and $\eta=0$.

(II) $\Rightarrow(\mathrm{III})$ is trivial, since $\widehat{F}(0)=1$. 
$(\mathrm{III}) \Rightarrow(\mathrm{I})$ : if $F$ is not aperiodic, then there exist $m \in\{2,3,4, \ldots\}$ and $r \in$ $\{0,1, \ldots, m-1\}$ such that $\operatorname{supp}(F) \subseteq m \mathbb{Z}+r$. But $r \neq 0$ since $F$ is adapted. Then

$$
\widehat{F}(2 \pi / m)=\sum_{l \in \mathbb{Z}} F(m l+r) e^{-i(m l+r) 2 \pi / m}=e^{-2 \pi i r / m} \notin \mathbb{D} \cup\{1\},
$$

and condition (III) fails.

$(\mathrm{IV}) \Rightarrow(\mathrm{III})$ : power-boundedness of $G$ implies (recall (15) ) that $\{\widehat{G}(\xi): \xi \in[-\pi, \pi]\}$ $\subseteq \overline{\mathbb{D}}$. Then $\widehat{F}(\xi)=\beta \widehat{G}(\xi)+(1-\beta) \in \mathbb{D} \cup\{1\}$ for all $\xi \in[-\pi, \pi]$.

To prove $(\mathrm{I}) \Rightarrow(\mathrm{IV})$, we begin with the special case where $F$ has a finite second moment.

Lemma 7.1. If $F \in \mathbb{P}(\mathbb{Z})$ is aperiodic and $\sum_{k \in \mathbb{Z}} k^{2} F(k)<\infty$, then $F$ satisfies condition (IV) of Proposition 2.5.

Proof of Lemma 7.1, Set $a:=\sum_{k \in \mathbb{Z}} k F(k) \in \mathbb{R}$. For each $\beta>0$ define $F_{\beta} \in L^{1}(\mathbb{Z})$ and $\tau_{\beta} \in C([-\pi, \pi])$ by

$$
F_{\beta}:=\beta^{-1}\left(F-(1-\beta) \delta_{0}\right), \quad \tau_{\beta}(\xi):=e^{i \beta^{-1} a \xi} \widehat{F}_{\beta}(\xi)=\sum_{k \in \mathbb{Z}} F_{\beta}(k) e^{-i\left(k-\beta^{-1} a\right) \xi}
$$

for $\xi \in[-\pi, \pi]$. Note that $F_{1}=F$. To obtain the lemma we must show that $F_{\beta}$ is power-bounded for some $\beta \in(0,1)$.

We first show that there exist $\beta_{0} \in(0,1)$ and $b>0$ such that

$$
\left|\tau_{\beta}(\xi)\right|=\left|\widehat{F}_{\beta}(\xi)\right| \leq 1-b \xi^{2}
$$

for all $\xi \in[-\pi, \pi]$ and $\beta \in\left(\beta_{0}, \beta_{0}^{-1}\right)$. Because $\sum_{k \in \mathbb{Z}} k^{2} F(k)<\infty$, one easily checks that $\tau_{\beta} \in C^{2}([-\pi, \pi])$ and that $\tau_{\beta}(0)=1, \tau_{\beta}^{\prime}(0)=0$ for all $\beta>0$. Thus Taylor's formula yields

$$
\tau_{\beta}(\xi)=1+\xi^{2} \int_{0}^{1} d s \tau_{\beta}^{\prime \prime}(s \xi)(1-s)
$$

for all $\xi \in[-\pi, \pi]$ and $\beta>0$. Observe that the functions $\tau_{\beta}^{\prime \prime}$ converge uniformly to $\tau_{1}^{\prime \prime}$ as $\beta \rightarrow 1$, and that $\tau_{1}^{\prime \prime}(0)=-\sum_{k \in \mathbb{Z}}(k-a)^{2} F(k)<0$. Then one deduces from (29) that there is a $\delta \in(0,1)$ such that an estimate (28) holds for all $\xi \in[-\delta, \delta]$ when $\beta$ is sufficiently close to 1 . Aperiodicity of $F$ implies that

$$
\sup \{|\widehat{F}(\xi)|: \xi \in[-\pi,-\delta] \cup[\delta, \pi]\}<1,
$$

so it is also easy to obtain an estimate of type (28) when $\xi \in[-\pi,-\delta] \cup[\delta, \pi]$ and $\beta$ is close to 1 . Thus (28) is obtained for some $\beta_{0} \in(0,1)$.

For the rest of the proof, fix $\beta \in\left(\beta_{0}, 1\right)$ and put $G:=F_{\beta}$. The Plancherel formula gives $\sum_{k \in \mathbb{Z}}|H(k)|^{2}=(2 \pi)^{-1} \int_{-\pi}^{\pi} d \xi|\widehat{H}(\xi)|^{2}$ for all $H \in L^{1}(\mathbb{Z})$. Using (28) and the inequality $1-b \xi^{2} \leq e^{-b \xi^{2}}$, we obtain an estimate

$$
\begin{aligned}
\sum_{k \in \mathbb{Z}}\left|G^{(n)}(k)\right|^{2} & =(2 \pi)^{-1} \int_{-\pi}^{\pi} d \xi\left|\left(\widehat{F}_{\beta}(\xi)\right)^{n}\right|^{2} \\
& \leq(2 \pi)^{-1} \int_{-\pi}^{\pi} d \xi e^{-2 b n \xi^{2}} \leq c n^{-1 / 2}
\end{aligned}
$$


for all $n \in \mathbb{N}$. Writing $\partial_{\xi}$ for differentiation with respect to $\xi$, observe that the $\mathbb{Z}$-Fourier transform of $k \mapsto-i\left(k-\beta^{-1}\right.$ an $) G^{(n)}(k)$ is

$$
\begin{aligned}
\left(\partial_{\xi}+i \beta^{-1} a n\right)\left(\widehat{F}_{\beta}(\xi)^{n}\right) & =e^{-i \beta^{-1} a n \xi} \partial_{\xi}\left(\tau_{\beta}(\xi)^{n}\right) \\
& =e^{-i \beta^{-1} a n \xi} n \tau_{\beta}(\xi)^{n-1} \tau_{\beta}^{\prime}(\xi) .
\end{aligned}
$$

Because $\tau_{\beta}$ is $C^{2}$ and $\tau_{\beta}^{\prime}(0)=0$, there is an estimate

$$
\left|\tau_{\beta}^{\prime}(\xi)\right|=\left|\tau_{\beta}^{\prime}(\xi)-\tau_{\beta}^{\prime}(0)\right| \leq c^{\prime}|\xi|, \quad \xi \in[-\pi, \pi] .
$$

By these facts and (28),

$$
\begin{aligned}
\sum_{k \in \mathbb{Z}}\left(k-\beta^{-1} a n\right)^{2}\left|G^{(n)}(k)\right|^{2} & =(2 \pi)^{-1} \int_{-\pi}^{\pi} d \xi\left|n \tau_{\beta}(\xi)^{n-1} \tau_{\beta}^{\prime}(\xi)\right|^{2} \\
& \leq c \int_{-\pi}^{\pi} d \xi n^{2} \xi^{2} e^{-2 b(n-1) \xi^{2}} \leq c^{\prime} n^{1 / 2}
\end{aligned}
$$

for all $n \in \mathbb{N}$. The Cauchy-Schwarz inequality, (30) and (31) imply that

$$
\begin{aligned}
& \sum_{k \in \mathbb{Z}}\left|G^{(n)}(k)\right| \\
& \leq\left(\sum_{k}\left[1+n^{-1}\left(k-\beta^{-1} a n\right)^{2}\right]^{-1}\right)^{1 / 2}\left(\sum_{k}\left[1+n^{-1}\left(k-\beta^{-1} a n\right)^{2}\right]\left|G^{(n)}(k)\right|^{2}\right)^{1 / 2} \\
& \quad \leq c n^{1 / 4}\left(\sum_{k}\left[1+n^{-1}\left(k-\beta^{-1} a n\right)^{2}\right]\left|G^{(n)}(k)\right|^{2}\right)^{1 / 2} \leq c^{\prime}
\end{aligned}
$$

for all $n \in \mathbb{N}$. Thus $G$ is power-bounded.

We next prove condition (IV) in case $F$ is aperiodic and $\sum_{k \in \mathbb{Z}} k^{2} F(k)=\infty$. In this case set $\gamma:=\sum_{k \in \mathbb{Z}}\left(1+k^{2}\right)^{-1} F(k) \in(0,1)$, and define $\widetilde{F} \in \mathbb{P}(\mathbb{Z})$ by

$$
\widetilde{F}(k)=\gamma^{-1}\left(1+k^{2}\right)^{-1} F(k), \quad k \in \mathbb{Z} .
$$

Since $\widetilde{F}$ is aperiodic and $\sum_{k \in \mathbb{Z}} k^{2} \widetilde{F}(k)<\infty$, by Lemma 7.1 there exist $\beta \in(0,1)$ and a power-bounded $\widetilde{G} \in L^{1}(\mathbb{Z})$ such that

$$
\widetilde{F}=\beta \widetilde{G}+(1-\beta) \delta_{0} .
$$

Because $F(k) \geq \gamma \widetilde{F}(k)$ for all $k \in \mathbb{Z}$, we have $F=\gamma \widetilde{F}+(1-\gamma) H$ for a certain $H \in \mathbb{P}(\mathbb{Z})$. Setting $\alpha:=1-\gamma+\beta \gamma \in(0,1)$ and

$$
G:=\frac{\beta \gamma}{\alpha} \widetilde{G}+\frac{1-\gamma}{\alpha} H \in L^{1}(\mathbb{Z}),
$$

it follows that $F=\alpha G+(1-\alpha) \delta_{0}$. Since $G$ is a convex combination of the powerbounded elements $\widetilde{G}, H$ in the (commutative) Banach algebra $L^{1}(\mathbb{Z})$, it is easy to see that $G$ is power-bounded. Thus $F$ satisfies condition (IV).

To complete the proof of Proposition 2.5, we verify (8). Let $F$ be aperiodic, let $G$ be as in condition (IV), and apply Theorem 2.3 with the operators $S:=L(F)$ and $T:=L(G)$ acting in $X=L^{1}(\mathbb{Z})$. Then (8) follows. 
Remark. One can prove (8) directly without using Theorem 2.3, at least in the case $\sum_{k \in \mathbb{Z}} k^{2} F(k)<\infty$. For in that case, by arguing in a similar way to the proof of Lemma [7.1] one can show that

$$
\sum_{k \in \mathbb{Z}}\left[1+n^{-1}(k-a n)^{2}\right]\left|F^{(n)}(k)-F^{(n+1)}(k)\right|^{2} \leq c n^{-3 / 2}
$$

for all $n \in \mathbb{N}$, where $a:=\sum_{k \in \mathbb{Z}} k F(k)$. Then (8) follows by the Cauchy-Schwarz inequality as in the proof of Lemma 7.1 .

\section{REFERENCES}

[1] C. Berg, K. Boyadzhiev and R. deLaubenfels, Generation of generators of holomorphic semigroups, J. Austral. Math. Soc. 55 (1993), 246-269. MR.1232759 (94g:47020)

[2] S. Blunck, Maximal regularity of discrete and continuous time evolution equations, Studia Math. 146 (2001), 157-176. MR.1853519 (2002g:34125)

[3] S. Blunck, Analyticity and discrete maximal regularity on $L_{p}$-spaces, J. Funct. Anal. 183 (2001), 211-230. MR1837537(2002c:47064)

[4] A. S. Carasso and T. Kato, On subordinated holomorphic semigroups, Trans. Amer. Math. Soc. 327 (1991), 867-878. MR1018572 (92a:47047)

[5] E. B. Davies, One-parameter semigroups. London Mathematical Society Monographs, vol. 15, Academic Press, London, 1980. MR.591851 (82i:47060)

[6] R. G. Douglas, Banach algebra techniques in operator theory. Second edition, Springer, New York, 1998. MR1634900 (99c:47001)

[7] N. Dungey, Time regularity for random walks on locally compact groups, Probab. Theory Relat. Fields 137 (2007), 429-442. MR2278463 (2007j:60008)

[8] N. Dungey, On time regularity and related conditions for power-bounded operators, Proc. London Math. Soc. (3) 97 (2008), 97-116. MR2434092

[9] A. Erdélyi, W. Magnus, F. Oberhettinger and F. G. Tricomi, Higher transcendental functions, volume I. McGraw-Hill, New York, 1953. MR0058756 (15:419i)

[10] W. Feller, An introduction to probability theory and its applications, volume II. John Wiley and Sons, New York, 1966. MR0210154 (35:1048)

[11] S. R. Foguel and B. Weiss, On convex power series of a conservative Markov operator, Proc. Amer. Math. Soc. 38 (1973), 325-330. MR0313476 (47:2030)

[12] G. B. Folland, A course in abstract harmonic analysis. CRC Press, Boca Raton, 1995. MR:1397028 (98c:43001)

[13] J. Galambos, Advanced probability theory. Second edition, Marcel Dekker, New York, 1995. MR.1350792 (96k:60002)

[14] M. Haase, The functional calculus for sectorial operators. Birkhäuser Verlag, Basel, 2006. MR2244037 (2007j:47030)

[15] P. R. Halmos, A Hilbert space problem book. Van Nostrand, Princeton, 1967. MR.0208368 $(34: 8178)$

[16] N. Kalton, S. Montgomery-Smith, K. Oleszkiewicz and Y. Tomilov, Power-bounded operators and related norm estimates, J. London Math. Soc. 70 (2004), 463-478. MR2078905 (2005e:47020)

[17] T. Kato, Note on fractional powers of linear operators, Proc. Japan Acad. 36 (1960), 94-96. MR0121666 (22:12400)

[18] T. Kato, Perturbation theory for linear operators. second edition, Grundlehren der mathematischen Wissenschaften 132, Springer-Verlag, 1976. MR0407617 (53:11389)

[19] Yu. Lyubich, The single-point spectrum operators satisfying Ritt's resolvent condition, Studia Math. 145 (2001), 135-142. MR1828001 (2002c:47070)

[20] C. Martínez Carracedo and M. Sanz Alix, The theory of fractional powers of operators. North-Holland Mathematics Studies 187, North-Holland Publishing Co., Amsterdam, 2001. MR.1850825 (2002k:47038)

[21] A. Montes-Rodríguez, J. Sánchez-Álvarez and J. Zemánek, Uniform Abel-Kreiss boundedness and the extremal behaviour of the Volterra operator, Proc. London Math. Soc. 91 (2005), 761788. MR2180462 (2006j:47053) 
[22] O. Nevanlinna, Convergence of iterations for linear equations. Birkhäuser, Basel, 1993. MR:1217705 (94h:65055)

[23] O. Nevanlinna, On the growth of the resolvent operators for power bounded operators, Linear operators (eds. J. Janas, F. H. Szafraniec and J. Zemánek), Banach Center Publication 38 (Institute of Mathematics of the Polish Academy of Sciences, 1997), pp. 247-264. MR1457011 (98e:47006)

[24] O. Nevanlinna, Resolvent conditions and powers of operators, Studia Math. 145 (2001), 113134. MR.1828000 (2002d:47004)

[25] W. Rudin, Real and complex analysis. Third edition, McGraw-Hill, 1987. MR924157 (88k:00002)

[26] F. Spitzer, Principles of random walk. Second edition, Springer-Verlag, New York, 1976. MR0388547 (52:9383)

[27] P. Vitse, Functional calculus under the Tadmor-Ritt condition, and free interpolation by polynomials of a given degree, J. Funct. Anal. 210 (2004), 43-72. MR2051632 (2005f:47046)

[28] K. Yosida, Functional Analysis. Grundlehren der mathematischen Wissenschaften 123, Springer-Verlag, 1980. MR617913 (82i:46002)

Department of Mathematics, Macquarie University, NSW 2109, Australia 